\title{
Relato de experiência sobre educação em saúde na escola: alimentação saudável e higiene pessoal
}

\author{
Experience report on health education at school: healthy eating and personal hygiene \\ Informe de experiencia sobre educacíon sanitária en La escuela: alimentación saludable e higiene \\ personal
}

\section{Gabrielle Silva Nascimento ${ }^{1 *}$, João Victor Manço Resende ${ }^{2}$, Luanna Barci Dutra da Costa ${ }^{3}$, Jorge Luiz Lima da Silva ${ }^{4}$, Larissa Murta Abreu ${ }^{5}$, Giulia Lemos de Almeida ${ }^{6}$}

Como citar esse artigo. Nascimento, GS; Resende, JVM; da Costa, LBD; da Silva, LL; Abreu, LM; de Almeida, GL. Relato de experiência sobre educação em saúde na escola: alimentação saudável e higiene pessoal. Revista Pró-UniverSUS. 2020 Jul./Dez.; 11 (2): 180 - 183.

\section{Resumo}

Introdução: o estudo tem por objetivo central descrever as vivências dos acadêmicos de enfermagem, durante o ensino teóricoprático da disciplina de saúde coletiva, explorando as contribuições das atividades de educação em saúde desenvolvidas no ambiente escolar. Materiais e métodos: estudo descritivo que se deu por meio de relato de experiência. A ação teve por finalidade abordar as temáticas sobre alimentação saudável e higiene pessoal com alunos do ensino fundamental. O público alvo foram crianças de oito a doze anos e as atividades ocorreram no primeiro trimestre de 2019, em três etapas. A primeira voltada para ambientação no cenário escolar, e reuniões externas para a elaboração do material e as duas últimas destinadas para a realização das atividades. Resultados: foram utilizadas técnicas de metodologias ativas, como quadro interativo e gincana. Na atividade de alimentação, foi identificado o grau de conhecimento sobre hábitos saudáveis das crianças. Com relação à higiene pessoal, foi perceptível o interesse e o aparecimento de dúvidas sobre o tema. Discussão: foi observada a importância da educação em saúde no ambiente escolar, dada a influência positiva do incentivo às práticas alimentares saudáveis e manutenção dos hábitos de higiene, a partir da utilização recursos lúdicos para adaptação da realidade. Conclusão: além de receberem informações, os alunos tiveram potencial de serem replicadores dos conhecimentos adquiridos nessas atividades, seja para suas famílias ou para a sociedade.

Palavras-chave: Educação Em Saúde; Alimentação Escolar; Higiene; Saúde Pública.

Afiliação dos autores:

${ }^{1}$ Acadêmica de enfermagem. Universidade Federal Fluminense/ Niterói/ Rio de Janeiro/ Brasil. E-mail: silvagabrielle@id.uff.br ORCID: https://orcid.org/0000-0002-4194-226X ${ }^{2}$ Acadêmico de enfermagem. Universidade Federal Fluminense/ Niterói/ Rio de Janeiro/ Brasil. E-mail: jmanco@id.uff.br ORCID: https://orcid.org/0000-0001-7534-3831

${ }^{3}$ Acadêmica de enfermagem. Universidade Federal Fluminense/ Niterói/ Rio de Janeiro/ Brasil. E-mail: luannabarci@id.uff.br ORCID: https://orcid.org/0000-0003-0296-8667 ${ }^{4}$ Docente. Universidade Federal Fluminense/Niterói/ Rio de Janeiro/ Brasil. E-mail: jorgeluizlima@gmail.com ORCID: https://orcid.org/0000-0002-2370-6343

${ }^{5}$ Acadêmica de enfermagem. Universidade Federal Fluminense/ Niterói/ Rio de Janeiro/ Brasil. E-mail: larissamurtaabreu9@gmail.com ORCID: https://orcid.org/0000-0002-71194370

${ }^{6}$ Acadêmica de enfermagem. Universidade Federal Fluminense/ Niterói/ Rio de Janeiro/ Brasil. E-mail: giulialemos@id.uff.br ORCID: https://orcid.org/0000-0003-1783-3298 


\section{Resumen}

Introducción: el objetivo principal del estudio es describir las experiencias de los estudiantes de enfermería, durante la enseñanza teórico-práctica de la disciplina de salud colectiva, explorando las contribuciones de las actividades de educación para la salud desarrolladas en el entorno escolar. Materiales y métodos: un estudio descriptivo que se realizó a través de un informe de experiencia. La acción tuvo como objetivo abordar los temas de alimentación saludable e higiene personal con estudiantes de primaria. El público objetivo eran niños de ocho a doce años y las actividades tuvieron lugar en el primer trimestre de 2019 , en tres etapas. La primera se centró en la configuración de la escena escolar y las reuniones externas para preparar el material y las dos últimas tuvieron como objetivo llevar a cabo actividades. Resultados: se utilizaron técnicas de metodologías activas, como tablero interactivo y gymkhana. En la actividad de alimentación, se identificó el grado de conocimiento sobre hábitos saludables de los niños. Con respecto a la higiene personal, el interés y la aparición de dudas sobre el tema fueron notables. Discusión: se observó la importancia de la educación para la salud en el entorno escolar, dada la influencia positiva de fomentar prácticas de alimentación saludable y mantener hábitos de higiene, utilizando recursos recreativos para adaptarse a la realidad. Conclusión: además de recibir información, los estudiantes tenían el potencial de ser replicadores del conocimiento adquirido en estas actividades, tanto para sus familias como para la sociedad

Palabras clave: Educación En Salud; Alimentación Escolar; Higiene; Salud Pública.

\section{Introdução}

No Brasil, para corroborar com os pensamentos acerca da educação em saúde, tendo como foco os estudantes, possibilitando a integralidade de diferentes setores da sociedade, foi criado através do Decreto $\mathrm{n}^{\circ}$ 6.286, de 5 de dezembro de 2007 o Programa de Saúde na Escola - PSE, que colocava em prática ações de promoção à saúde e prevenção ${ }^{1}$.

O PSE representa um elo entre a educação e o âmbito da saúde, uma vez que ambos devem caminhar juntos para garantir qualidade e integralidade aos estudantes ${ }^{2}$.

Nesse sentido, é possível proporcionar, por meio da educação, o conhecimento sobre a prevenção e promoção da saúde de crianças de faixa etária de 7 a 10 anos, que, considerando suas fases, âmbito social e físico, merecem atenção e métodos as possibilitem entender a importância de uma alimentação saudável e higiene corporal adequada ${ }^{3}$.

Dentre as atividades a serem realizadas pela equipe de saúde, a educação em saúde é primordial. Desta forma, os profissionais devem adquirir as habilidades e competências necessárias para disseminar e informar práticas saudáveis e preventivas, influenciando os indivíduos a adquirir novos hábitos de vida ${ }^{4}$.

$\mathrm{O}$ artigo tem por objetivo descrever as vivências dos acadêmicos de enfermagem durante o ensino teórico-prático da disciplina de saúde coletiva ao longo da atividade curricular em campo prático, com vistas a aprimorar os conceitos apreendidos e embasar as constatações feitas pelo grupo após a experiência com a educação em saúde.

\section{Material e Método}

Estudo exploratório com abordagem descritiva, desenvolvido por meio relato de experiência realizado em um colégio federal. A elaboração e execução na instituição de ensino ocorreram durante o primeiro semestre de 2019, por oito acadêmicos do quarto período do curso de graduação em enfermagem.

O colégio atende a turmas de educação infantil e básica, sendo as atividades promovidas, em campo, para alunos do terceiro, quarto e quinto ano do ensino fundamental. As práticas foram supervisionadas por professores e monitores e os temas abordados pelo grupo foram: higiene pessoal e práticas alimentares saudáveis.

Os eixos temáticos foram previamente escolhidos pelos alunos, com idades de oito a doze anos, na presença de seus professores e auxiliares, visando à horizontalidade, à compreensão de todos, e a possibilidade de maior interação e aprendizado por todas as partes envolvidas. Com isso, o projeto consistiu em elaborar exposições, estabelecidas pela coordenadora pedagógica e por professores, de modo a estimular o convívio com os alunos para que as mesmas fossem interessantes e instigassem a participação.

Os procedimentos que envolveram a experiência foram realizados em três etapas, sendo o primeiro momento voltado para ambientação, reuniões, e para a elaboração do material apresentado para as crianças. As duas últimas visitas ao colégio, foram destinadas à realização das atividades. Foram utilizadas técnicas de metodologias ativas para atrair a atenção dos alunos e promover o entendimento por meio ludicidade.

\section{Resultados}

Diante da situação apresentada, foi decidido debater com os alunos sobre temas como higiene, abordando mitos e verdades sobre a alimentação intradomiciliar e na escola, na tentativa de identificar os alimentos saudáveis e não saudáveis, além de realizar uma pesquisa sobre o que os alunos gostariam de comer no colégio.

Após a seleção das temáticas, foi acordado que, para atrair a atenção dos alunos, a faixa etária deveria ser levada em consideração. Com isso, optou-se por uma apresentação dinâmica, na qual o conteúdo teórico seria apresentado, de formulação simples para facilitar a 
compreensão dos alunos envolvidos na dinâmica. Nesse sentido, com a finalidade de estabelecer abordagem lúdica, foram utilizadas ferramentas interativas, com o auxílio de quadro participativo e gincanas, elaboradas anteriormente pelos acadêmicos.

Para a primeira atividade sobre alimentação, o grupo que contava com sete estagiários, preparou um quadro interativo com dois lados, sendo um deles com os alimentos saudáveis e outro com aqueles considerados "não saudáveis". O grupo dispunha de imagens aleatórias de alimentos variados e puderam correlacioná-las aos seus respectivos lados de acordo com suas próprias concepções acerca da temática da alimentação.

Finalizada a etapa anterior, os alunos receberam uma folha de papel, contendo o desenho de um prato e foram incumbidos a desenhar o que gostariam de comer no colégio. Essa atividade foi solicitada pelas professoras do colégio, uma vez que os alunos se recusavam a comer no refeitório, além de substituírem a refeição da escola por guloseimas que levavam nas mochilas, o que é expressamente proibido de acordo com o regulamento do colégio.

Em todas as turmas, os alimentos citados no quadro interativo na coluna "saudável" foram bem semelhantes, sendo eles: ovo, peixe e outras variedades de feijão, além de mais opções de frutas como uva, morango e pêra. Durante a dinâmica, notou-se que havia um aluno que possui dieta vegana e relatou que não encontrava no colégio opções variadas de alimentação, e outro aluno que comia as frutas durante o almoço por não gostar do sabor das refeições.

Para a atividade sobre higiene pessoal, os estagiários arrecadaram diversos produtos como sabonete, creme dental e escova de dente, com o propósito de montar kits, a serem distribuídos para as crianças no final na gincana. Todos os produtos distribuídos foram obtidos com a colaboração da população e da comunidade acadêmica, a partir de postagens nas redes sociais.

Essa etapa contou com dinâmica proativa dos alunos, que em um primeiro momento foram divididos em três equipes: azul, verde e vermelho. Cada equipe correspondia a uma turma. A gincana se iniciou com uma série de perguntas de respostas rápidas abordando as práticas de higiene, intituladas de "fala sério?", ou "com certeza!". O objetivo era observar o conhecimento dos alunos sobre o assunto e instrui-los em aspectos necessários como a higiene bucal, lavagem das mãos e higiene íntima.

Por fim, a atividade uniu a diversão e a educação em saúde, onde os alunos deveriam encontrar no pátio do colégio imagens de objetos ou produtos de higiene pessoal, como uma espécie de caça ao tesouro, os quais foram previamente escondidos pelos estagiários.

Assim, nessa dinâmica sobre a higiene pessoal foi perceptível que as crianças possuíam conhecimento prévio sobre o assunto, pois respondiam de forma correta e explicativa as questões abordadas durante a gincana.

\section{Discussão}

O ambiente escolar se mostra propício ao estabelecimento de relações favoráveis à promoção da saúde e a práticas de prevenção de doenças e agravos de saúde por se estabelecer o convívio social ${ }^{1}$.

Nessa concepção, a educação em saúde torna-se um instrumento fundamentalmente ativo nos processos transformativos do indivíduo, trabalhando a autonomia dos sujeitos e os impulsionando a desenvolverem habilidades pessoais, através de métodos educativos para propagação do conhecimento da saúde, voltados para o reconhecimento de comportamentos e hábitos de risco $^{4}$.

O ambiente escolar torna-se propício para a formação de uma maneira mais saudável de vida do estudante, perpassando desde os padrões cognitivos até os padrões culturais, comportamentais e sociais do indivíduo. Sendo assim, o cenário da escola obtém fatores predisponentes levando a ser um ambiente privilegiado para atingir a promoção de saúde de modo ampliado, visando à construção de cidadania ${ }^{5}$.

No Brasil, desde o Decreto $\mathrm{N}^{\circ} 7.272$, de 25 de agosto de 2010 foi estabelecido a Educação Alimentar e Nutricional (EAN) como uma diretriz da Política Nacional Alimentar e Nutricional (PNSAN), ampliando sua contribuição no crescimento e desenvolvimento de ações de educação alimentar e nutricionais para a formação de hábitos saudáveis dos estudantes, além da oferta de refeições durante o período letivo ${ }^{3,6}$.

De acordo com os resultados obtidos nas atividades propostas, nota-se a relevância no direcionamento adequado da alimentação infantil. Contudo, apesar do colégio seguir as normas de oferta de refeições, foram observadas algumas resistências, por parte dos alunos, no que tange a frequência do consumo de merenda escolar, demonstrando que os mesmos tinham insatisfações no preparo de certos alimentos quando as expressões "feijão aguado" e "arroz sem sabor" foram citadas por muitos.

Com isso, as preparações do cardápio devem ser reformuladas tendo em vista a percepção dos escolares, assim como suas preferências e suas atitudes em relação à alimentação escolar nas propostas educativas?

Devido a esses fatores, somados ainda à problemática crescente da obesidade infantil e as demais doenças que podem ser ocasionadas por uma alimentação desregulada e pobre em nutrientes é necessário que haja mobilização e incentivos que fomentem as práticas alimentares mais saudáveis dentro e fora das escolas ${ }^{8,9}$. 
A importância da educação em saúde no campo nutricional no ambiente escolar é essencial para consolidar hábitos alimentares saudáveis, permitindo mudanças no padrão de vida e evitando a elevação das taxas de morbidade e mortalidade por essas causas. Nota-se a necessidade da ampliação dessas ações não apenas os escolares, mas atingindo também a rede familiar $^{8,9}$.

Analisando os resultados, também se sugere que através do ensino lúdico a aprendizagem torna-se mais atrativa ao público infantil. Sobre a aprendizagem por meio de jogos, torna-se evidente que as regras ajudam as crianças com relação à sociedade, melhorando seu convívio com os demais, auxiliando na aprendizagem, além de serem prazerosas ${ }^{10}$.

Nesse sentido, analisando as crianças como seres atemporais, deve-se trabalhar a educação em saúde a partir do que está em evidência na atualidade. Tanto as práticas lúdicas, quanto a interação por meio das redes sociais podem ser instrumentos para a capacitação para o autocuidado e disseminação da educação em saúde que deve iniciar-se na primeira infância.

Sendo assim, a integralidade de diferentes setores da sociedade, como por exemplo, a família, a rede de saúde e, principalmente, as escolas e os diferentes segmentos de atuação dentro dela, constitui um processo de mudança capaz de instruir, orientar e principalmente causar uma modificação de posicionamento e postura através de uma reflexão crítica dos temas abordados.

\section{Considerações finais}

A saúde coletiva visa promover a qualidade de vida da população em geral, de forma a aplicar conhecimentos técnicos e científicos, a desenvolver a saúde de modo a assistir o ser humano de forma completa. Nesse sentido, se tratando de crianças em idade escolar, observa-se a necessidade da integração e estreitamento dos laços entre a universidade e as unidades escolares para melhor qualidade de assistência aos estudantes.

Em vista disso, o conteúdo explicitado neste artigo destacou a importância da participação dos acadêmicos de enfermagem ao proporem atividades diversificadas abordando os diversos temas que permeiam a educação em saúde. Logo ao atuarem como educadores podem se tornar importantes instrutores para alertar sobre hábitos nocivos à saúde das crianças ou em relação à alimentação saudável e higiene pessoal.

Após a adaptação do conteúdo ao público em questão, além de meramente receberem informações, os alunos tiveram potencial de atuar na construção do próprio conhecimento, sendo importantes replicadores das informações adquiridas durante as atividades, seja para suas famílias e também para sociedade.

\section{Referências}

1. Brasil. Ministério da Educação. Programa saúde nas Escolas. Brasil, 2018. Disponível em: http://portal.mec.gov.br/expansao-da-rede-federal/194secretarias-112877938/secad-educacao-continuada-223369541/14578programa-saude-nas-escolas. Acesso em: junho, 2019.

2. Brasil. Ministério da Saúde. Secretaria de Atenção à Saúde. Departamento de Atenção Básica. Saúde na escola. Brasília: Ministério da Saúde, Cadernos de Atenção Básica; n. 24, 2009, p. 96.

3. Brasil. Ministério da Educação. Programa Nacional de Alimentação Escolar- PNAE: Educação Alimentar e Nutricional - EAN. Disponível em: http://www.fnde.gov.br/programas/pnae/pnae-eixos-de atuacao/ pnaeeducacao alimentarnutricional?highlight $=$ WyJlc2NvbGEiXQ $==\& g t$. Acesso em: julho, 2019.

4. Ferreira ADS, Costa EFL, Farias KF, Bezerra RP, Dantas TF, Zacarias VS. A história da educação em saúde e seus modelos de prática impostos à sociedade. Santana do Ipanema/AL: diversitas journal, 2016. Disponível em: https://periodicos.ifal.edu.br/diversitas_journal/article/view/379. Acesso em: outubro, 2019

5. Couto NA, Kleinpaul WV, Borfe L, Vargas SC, Pohl HH, Krug SF. $\mathrm{O}$ ambiente escolar e as ações de promoção da saúde. Santa Cruz do Sul: Cinergis 2016.

6. Brasil. Decreto $\mathrm{n}^{\circ} 7.272$, de 25 de agosto de 2010. Regulamenta a Lei no 11.346, de 15 de setembro de 2006, que cria o Sistema Nacional de Segurança Alimentar e Nutricional - SISAN. Diário Oficial da União, 2010

7. Greenwood RL, Fonseca AB. Alimentação na escola: significados e possibilidades do comer e da comida para a promoção dos direitos humanos. Revista Interdisciplinar de Direitos Humanos, 2018; 5(1):103-127. Disponível em: <https://www3.faac.unesp.br/ridh/index.php/ridh/article/ view/572/241>. Acesso em: novembro, 2019.

8. Organização Mundial da Saúde. Plano de Ação para Prevenção da Obesidade em Crianças e Adolescentes. Washington, D.C., USA, 2014. Disponível em: https://www.paho.org/bra/images/stories/UTFGCV/ planofactionchildobesitypor.pdf?ua=1. Acesso em: junho, 2019.

9. Lopes CAO, Brant ER, Coelho LSVA, Santiago SSS, Romano MCC. Prevenção da obesidade infantil: uma proposta educativa. In: Interfaces Revista de Extensão da UFMG. Belo Horizonte, 2019. Disponível em: $<$ https://www.ufmg.br/proex/revistainterfaces/index.php/IREXT/article/ view/28/pdf>. Acesso em: outubro, 2019.

10. Silva VMVBM. Importância do lúdico no ensino de higiene para alunos do ensino fundamental: utilização de jogo da memória. Revista F@ pciência, Apucarana-PR, 2014;10(1):31-39. Disponível em: http://www. cesuap.edu.br/fap-ciencia/edicao_2014/006.pdf. Acesso em: junho, 2019.

11. Cunha LF. A importância de uma alimentação adequada na educação infantil. Ibaiti, 2014. Disponível em: http://repositorio.roca.utfpr.edu.br/ jspui/bitstream/1/3507/1/MD_ENSCIE_IV_2014_57.pdf. Acesso em: junho, 2019.

12. Ministério da Saúde. Dez passos para uma alimentação saudável. Guia alimentar para crianças menores de dois anos. Ministério da Saúde, Secretaria de Atenção à Saúde, Departamento de Atenção Básica. - 2 ed. - 2 reimpr. - Brasília - DF: Ministério da Saúde, 2013.

13. Moril WMR. Metodologias de ensino-aprendizagem na promoção da educação em saúde. Brasília, artigo de conclusão de curso de pósgraduação da Faculdade de Ciências de Wenceslau Braz (FACIBRA), Julho, 2013. Disponível em; https://semanaacademica.org.br/system/files/artigos/ metodologias_de_ensinoaprendizagem_na_promfoouo_da_educaouo_em sade. orientaouo educacional_0.pdf. Acesso em: julho, 2019.

14. Política Nacional de Alimentação e Nutrição, 2013, $1^{\text {a }}$ edição Disponível em: http://bvsms.saude.gov.br/bvs/publicacoes/politica nacional_alimentacao_nutricao.pdf. Acesso em: junho, 2019.

15. Silva LFS, Alves NC. Higiene pessoal: a importância de estudar o corpo humano. Biodiversidade, 2014;13(2):75-89.

16. Vieira M, Vanin AC, Souza D, Piantino CB. Infância saudável: Educação em Saúde nas Escolas. 2017;[S.1.]. Disponível em: https:// periodicos.ufpel.edu.br/ojs $2 /$ index.php/expressaextensao/article/ view/10808/741. Acesso em: 21 de junho de 2019. 